Technical note

\title{
Novel horseradish peroxidase substrates for use in immunohistochemistry
}

\author{
Kenneth H. Petersen * \\ Dako A/S, Produktionsvej 42, DK-2600 Glostrup, Denmark
}

\section{A R T I C L E I N F O}

\section{Article history:}

Received 1 July 2008

Received in revised form 22 September 2008

Accepted 24 September 2008

Available online 6 November 2008

\section{Keywords:}

Cyanine dye

Chromogen

Horseradish peroxidase

Immunohistochemistry

Double stain

\begin{abstract}
A B S T R A C T
New chromogens expand the colour palette for horseradish peroxidase chromogens used in immunohistochemistry. Tissue staining of cytokeratin with three new cyanine-based chromogens is described. Their use as fluorescent reporters is demonstrated.
\end{abstract}

(c) 2008 Elsevier B.V. All rights reserved.

\section{Introduction}

Immunohistochemistry (IHC) is based on the use of chromogens to visualize different targets within tissues. The IHC visualization systems used are based on antibodies conjugated with an enzyme. The most common enzymes used in the conjugates are horseradish peroxidase (HRP) and alkaline phosphatase. Substrates of these enzymes form coloured precipitates when processed with the appropriate enzyme. For HRP, the common substrates are 3,3'-diaminobenzidine (DAB, brown), 3,3',5,5'-tetramethylbenzidine (turquoise), and 3-amino-9-ethyl carbazole (AEC, brown/red). A few modifications of these substrates are described in the literature for use in IHC, to yield different colours or improved staining properties (Hsu and Soban, 1982; Nemes, 1987; Krieg et al., 2000; Krieg and Halbhuber, 2003).

For fluorescent in situ hybridization (FISH), the most common enzyme substrate is the tyramide system, in which a fluorescent reporter is coupled to tyramine and used as the

Abbreviations: AEC, 3-amino-9-ethyl carbazole;DAB, 3,3'-diaminobenzidine;DAPI, 4',6-diamidino-2-phenylindole;FISH, Fluorescent in situ hybridization;IHC, Immunohistochemistry.

* Tel.: +45 44859500 .

E-mail address: Kenneth.Petersen@dako.com. substrate for HRP (Schmidt et al., 1997; Speel et al., 1999). Fluorescein or a cyanine dye is often used as the fluorescent reporter. Other fluorescent HRP substrates with self-anchoring properties have recently been described in the literature (Krieg et al., 2007, 2008).

Cyanine dyes, with their intense absorption band, are intensely coloured and were originally used for dyeing textiles. Their intense colours make them good candidates for new IHC chromogens. Today, cyanine dyes (Cy3, Сy5) are used as fluorochrome labels on biomolecules. This communication presents the novel application of cyanine dyes as peroxidase substrates, for use as either fluorochromes or chromogens.

\section{Experimental}

Dako IHC reagents were used throughout all experiments.

Stock solutions of the compounds in N-methylpyrrolidone (20-30 mg/mL) were stored at $5{ }^{\circ} \mathrm{C}$. For staining, these stock solutions were diluted in the DAB buffer component of the kits, thus substituting these compounds for DAB. The final concentration was $0.5 \mathrm{mg} / \mathrm{mL}$ when used as chromogens and $10 \mu \mathrm{g} / \mathrm{mL}$ for fluorescence applications.

The dewaxed tissue was antigen retrieved for $10 \mathrm{~min}$ in a microwave oven, washed in Tris-buffered saline (TBS), and incubated for $30 \mathrm{~min}$ with monoclonal mouse antibody directed against human cytokeratin. After washing, the tissue was 
incubated with EnVision ${ }^{+}$for 30 min, then washed again. The tissue was then stained with one of compounds I-III for $10 \mathrm{~min}$. The tissue was washed in TBS, counterstained with Nuclear Fast Red if needed, and mounted in Faramount for light microscopy or Vectashield with DAPI $(0.1 \mu \mathrm{g} / \mathrm{mL})$ for fluorescence microscopy. The fluorescence microscope was a Leica DMRA fitted with a camera and Chroma filter \#31000 (Cy5) or \#51006 (Cy3) or Omega Optical filter XF-114-2 (DAPI). The images were digital overlays of two pictures taken with different exposure times.

Double staining was performed with the Envision $\mathrm{G} \mid 2$ double staining system using cytokeratin diluted 1:50 and CD20 diluted 1:100. Cytokeratin was stained first and compound 1 was substituted for DAB in the substrate buffer. No counterstaining was used. The pictures were taken with an ACIS III Image Analysis System.

\section{Discussion}

If a compound contains an aromatic hydroxyl or amino group, it can be a substrate for horseradish peroxidase. Mujumdar et al. (1989) described cyanine dyes containing one amino group in each of their indole ring systems and (Shragina et al.,1990) described indoles containing one hydroxyl in their aromatic ring. The cyanine dyes shown in Fig. 1 were synthesized based on their methods.

The compounds were tested for IHC using AEC as the reference. Fig. 2A-D shows cytokeratin staining in colon tissue. The compounds form a blue or turquoise precipitate. Nuclear Fast Red was preferred for counterstaining, and methyl green can also be used, but hematoxylin is not suitable because it produces a blue counterstain. A twofold dilution series showed that the staining intensity of compound I was similar to that of AEC, whereas compounds II and III showed lower staining intensities. These staining intensities indicate that an amino group on each indole ring is better than a hydroxyl group for this purpose. The $\mathrm{Cy}-5$ analogue was therefore only synthesized with amino groups on the indole rings and only the aminobearing cyanines were tested as fluorescent substrates. Diluting the IHC substrates 50 times made it possible to use them as fluorescent substrates. Fig. $2 \mathrm{~F}$ shows the immunofluorescent staining of colon tissue with compound I as the precipitating fluorochrome.

A single concentration of each compound could not be used for both chromogenic and fluorescent applications. If the chromogen concentration was raised above $0.5 \mathrm{mg} / \mathrm{mL}$ for use as a chromogen or $10 \mu \mathrm{g} / \mathrm{mL}$ for fluorescent applications, then nonspecific staining of the tissues by the dyes was observed. When a chromogen stain was observed by fluorescence microscopy, the whole tissue was brightly fluorescent and no specific signals were seen.

The dyes are transformed into radicals by the action of HRP. These radicals can either react with the surrounding tissue forming covalent bonds (self-anchoring), or they can react with other dye molecules to form a polymer that precipitates. The product in both reactions is insoluble and the resultant stain is believed to be a mixture of the two reactions. The ratio of the two reaction products is expected to be concentration dependent. The polymerization of compound I was observed by the addition of HRP to a test tube containing the substrate solution. This caused instant precipitation and mass spectroscopic analysis of the solution showed dimers of compound I in the solution.

The reaction product is insoluble in both water and organic solvents. This confers the advantage that stained slides can be mounted in both water-based and permanent mounting media. For fluorescence applications, this means that fluorescence spectra of the insoluble precipitates are not possible and the filter sets were chosen based on the commercial Cy3 and Cy5 data.

\section{Conclusion}

Known fluorochromes have successfully been converted into substrates for HRP, eliminating the need for a tyramine anchor to connect the substrate functionality to the dye. The compounds described here are a new class of self-anchoring HRP substrates. Of the three dyes described, compound I is the most promising for IHC because it is similar to AEC in its staining and sensitivity. In IHC, these dyes offer new colour combinations in the double staining of tissue. To demonstrate this, compound I was used together with Fast Red to doubly stain cytokeratin and CD20, shown in Fig. 2E. The stability of the

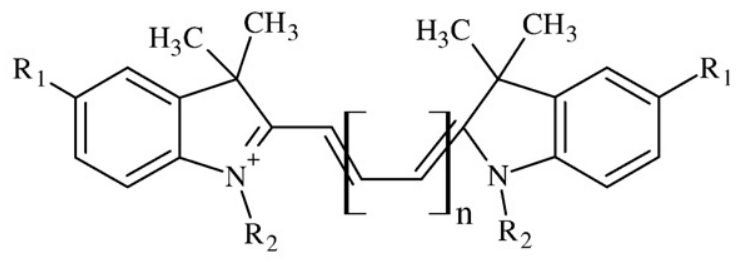

\begin{tabular}{c|ccccc} 
Compound & $\mathrm{n}$ & $\mathrm{R}_{1}$ & $\mathrm{R}_{2}$ & Colour & Counter ion \\
\hline I & 1 & $\mathrm{NH}_{2}$ & $\left(\mathrm{CH}_{2}\right)_{4} \mathrm{SO}_{3} \mathrm{H}$ & Blue & Trifluoroacetate \\
II & 1 & $\mathrm{OH}$ & $\mathrm{CH}_{2} \mathrm{CH}_{3}$ & Blue & Iodide \\
III & 2 & $\mathrm{NH}_{2}$ & $\left(\mathrm{CH}_{2}\right)_{4} \mathrm{SO}_{3} \mathrm{H}$ & Turquoise & Trifluoroacetate
\end{tabular}

Fig. 1. The cyanine dyes investigated. 
A

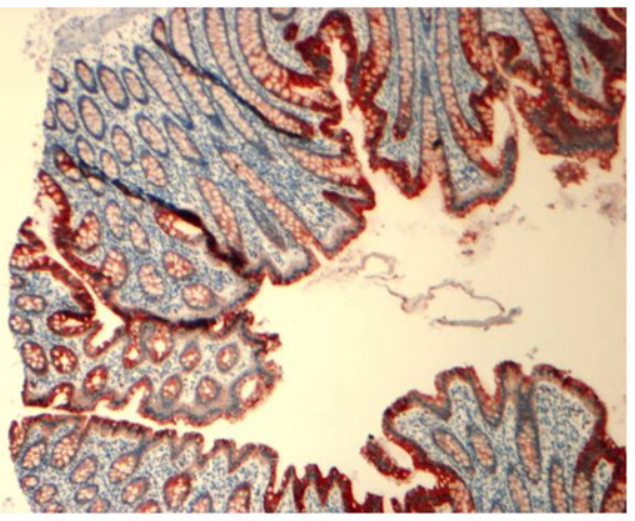

C

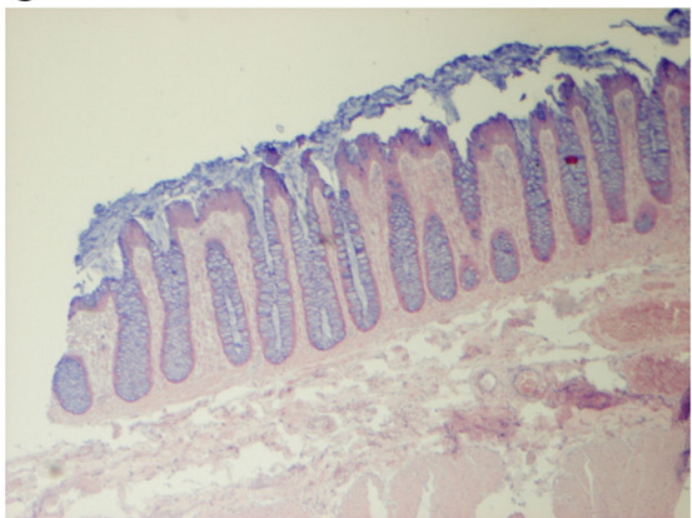

$\mathbf{E}$

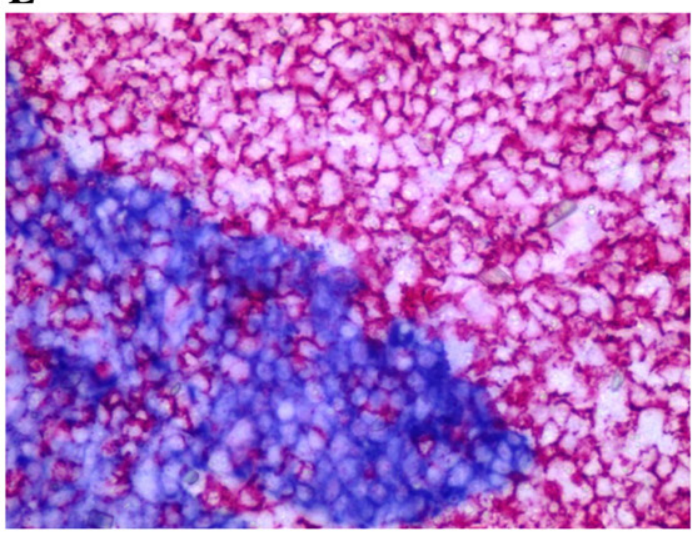

B

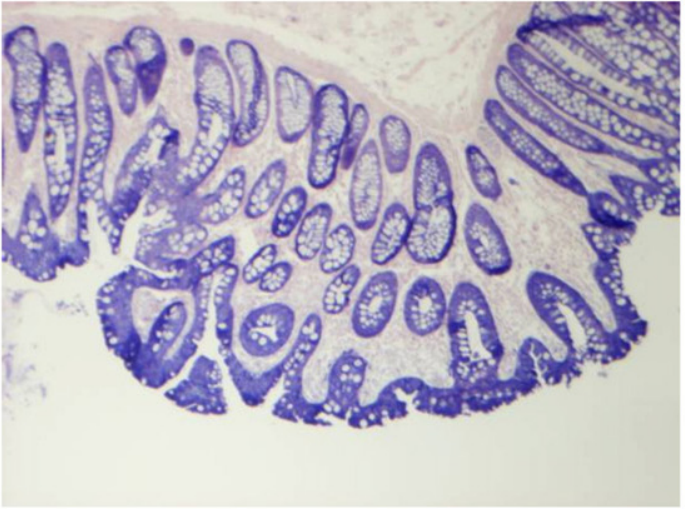

D

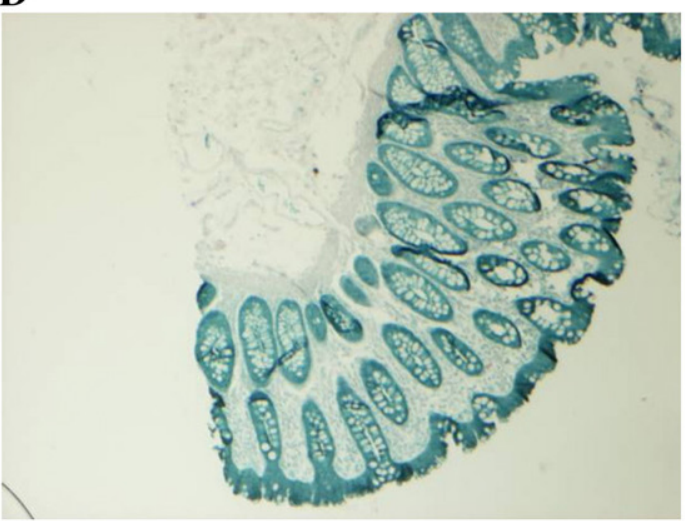

F

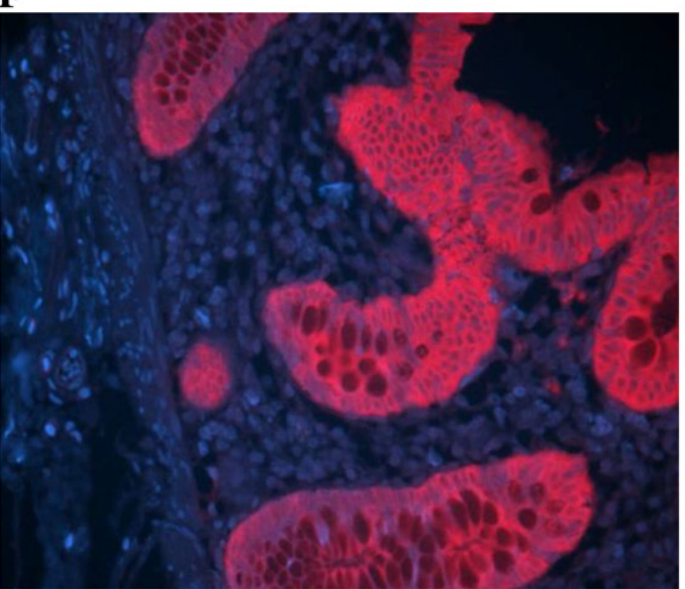

Fig. 2. A-D. Colon tissue stained with anti-cytokeratin/HRP. A-E. Light microscopy. F. Fluorescence microscopy. A. AEC/hematoxylin. B. Compound I/Nuclear Fast Red. C. Compound II/Nuclear Fast Red. D Compound III E. Tonsil stained with compound I (cytokeratin) and Fast Red (CD20). F. Colon tissue stained with compound I/DAPI.

chromogen-stained slides is good. Slides stored for more than 1 year can still be examined under a light microscope.

The compounds described here can be substituted directly into a normal DAB staining protocol, as a substitute for DAB.

\section{Acknowledgement}

I would like to thank Anne M. Maarbjerg for tissue staining.

\section{References}

Hsu, S.M., Soban, E., 1982. Color modification of diaminobenzidine (DAB) precipitation by metallic ions and its application for double immunohistochemistry. J. Histochem. Cytochem. 30, 1079.

Krieg, R., Halbhuber, K.J., 2003. Recent advances in catalytic peroxidase histochemistry. Cell. Mol. Biol. (Noisy-le-grand) 49, 547.

Krieg, R., Halbhuber, K.J., Oehring, H., 2000. Novel chromogenic substrates with metal chelating properties for the histochemical detection of peroxidasic activity, derived from 3-amino-9-ethylcarbazole (AEC) and 3,6-diamino-9ethylcarbazole. Cell. Mol. Biol. (Noisy-le-grand) 46, 1191. 
Krieg, R., Eitner, A., Günther, W., Halbhuber, K.-J., 2007. Optimization of heterocyclic 4-hydroxystyryl derivatives for histological localization of endogenous and immunobound peroxidase activity. Biotech. Histochem. 82, 235.

Krieg, R., Eitner, A., Günther, W., Schürer, C., Lindenau, J. and Halbhuber, K.-J. (2008) N,N-Dialkylaminostyryl dyes: specific and highly fluorescent substrates of peroxidase and their application in histochemistry, J. Mol. Histol. 39 (2) (Apr). 169.

Mujumdar, R.B., Ernst, L.A., Mujumdar, S.R., Waggoner, A.S., 1989. Cyanine dye labeling reagents containing isothiocyanate groups. Cytometry 10, 11.

Nemes, Z., 1987. Intensification of 3,3'-diaminobenzidine precipitation using the ferric ferricyanide reaction, and its application in the doubleimmunoperoxidase technique. Histochemistry 86, 415.
Schmidt, B.F., Chao, J., Zhu, Z., DeBiasio, R.L., Fisher, G., 1997. Signal amplification in the detection of single-copy DNA and RNA by enzymecatalyzed deposition (CARD) of the novel fluorescent reporter substrate Cy3.29-tyramide. J. Histochem. Cytochem. 45, 365.

Shragina, L., Buchholtz, F., Yitzchaik, S., Krongauz, V., 1990. Searching for photochromic liquid crystals spironaphthoxazine substituted with a mesogenic group. Liq. Cryst. 7, 643.

Speel, E.J., Hopman, A.H., Komminoth, P., 1999. Amplification methods to increase the sensitivity of in situ hybridization: play card(s). J. Histochem. Cytochem. 47, 281. 